\title{
Anatomia do sistema porta renal e suas implicações no emprego de agentes anestésicos na contenção de avestruzes (Struthio camelus)
}

\author{
Anatomy of the renal portal system and its implications for the use of anesthetic agents in the restraint of \\ ostriches (Struthio camelus)
}

\author{
Haley Silva de Carvalho ${ }^{\mathrm{I}}$ Rodrigo Ciboto ${ }^{\mathrm{II}}$ Camila Grinaboldi Baitelo ${ }^{\mathrm{III}}$ Ricardo Augusto Dias ${ }^{\mathrm{IV}}$ Silvia \\ Renata Gaido Cortopassi ${ }^{*}$
}

\section{RESUMO}

Objetivou-se com este estudo caracterizar a anatomia do sistema porta renal e verificar sua influência sobre o protocolo anestésico xilazina, tiletamina e zolazepam na contenção de avestruzes, por comparação da administração dos fármacos nos músculos da perna ou da asa. Em cinco animais foi injetado látex nas veias femorais no sentido de drenagem e, posteriormente, as aves foram fixadas em formol a 10\%, por 72 horas. Em uma ave, procedeu-se à localização, colheita e fixação das valvas portais renais em formol a $10 \%$. O sistema porta renal apresentou-se constituído por duas veias portais renais craniais, duas veias portais renais caudais e seis valvas portais renais. Na contenção química, seis avestruzes foram pré-tratados com xilazina $\left(1 \mathrm{mg} \mathrm{kg}^{-1}\right) \mathrm{e}$, decorridos 10 minutos, receberam tiletamina/zolazepam $\left(6 \mathrm{mg} \mathrm{kg}^{-1}\right)$. Os animais foram manipulados em duas ocasiões diferentes, sendo que na primeira anestesia o protocolo foi administrado nos músculos da base das asas (GI) e, após 15 dias, os mesmos animais receberam o protocolo nos músculos das pernas (GII). Os períodos de latência, hábil e de recuperação não foram diferentes entre os grupos $(P>0,05)$. A freqüência cardíaca permaneceu abaixo dos valores basais durante a anestesia $(P<0,05)$ nos dois grupos. A temperatura cloacal aumentou nos grupos, principalmente no GII, levando ao incremento da freqüencia respiratória para facilitar a perda de calor. Como a contenção química foi adequada para a realização de procedimentos de curta duração a campo nos avestruzes dos dois grupos, não foi possível evidenciar a influência do sistema porta renal.

Palavras-chave: anatomia, sistema porta renal, anestesia, avestruzes.

\begin{abstract}
This study was aimed at characterizing the anatomy of the renal portal system, and determining its influence on the anesthetic protocol xylazine, tiletamine and zolazepam, in the restraint of ostriches, compared with the administration of drugs into the leg or wing muscles. Latex was injected into the femoral veins of five animals, for drainage purposes, and the birds were then fixed in 10\% formaldehyde, for 72 hours. In one bird, the renal portal valves were located, collected and fixed in $10 \%$ formaldehyde. The renal portal system consisted of two cranial renal portal veins, two caudal renal portal veins, and six renal portal valves. In the chemical restraint, six ostriches were anesthetized with xylazine $\left(1 \mathrm{mg} \mathrm{kg}^{-1}\right)$ and after 10 minutes, tiletamine/zolazepam $\left(6 \mathrm{mg} \mathrm{kg}^{-1}\right)$. The animals were handled on two different occasions: in the first anesthesia, the protocol was administered into the muscles at the base of the wings (GI) and after 15 days, the same animals received the protocol in the leg muscles (GII). The periods for the onset and duration of the anesthesia, and recovery, showed no difference between the groups $(P>0.05)$. The heart rate remained below the basal values during the anesthesia $(P<0.05)$, in both groups. The cloacal temperature increased in both groups, particularly in GII, leading to an increased respiratory rate to facilitate heat loss. Since the chemical restraint was adapted for procedures of short duration in the field in the ostriches of both groups, it was not possible to demonstrate the influence of the renal portal system.
\end{abstract}

Key words: anatomy, renal portal system, anesthesia, ostriches.

IDepartamento de Cirurgia, Faculdade de Medicina Veterinária e Zootecnia (FMVZ), Universidade de São Paulo (USP). Av. Prof. Dr. Orlando Marques de Paiva, 87, Cidade Universitária, 05508-900, São Paulo, SP, Brasil. E-mail: silcorto@usp.br.*Autor para correspondência.

IIAutônomo, Osasco, SP, Brasil.

IIIAutônoma, Espírito Santo do Pinhal, SP, Brasil.

${ }^{\text {IV } D e p a r t a m e n t o ~ d e ~ M e d i c i n a ~ V e t e r i n a ́ r i a ~ P r e v e n t i v a ~ e ~ S a u ́ d e ~ A n i m a l, ~ F M V Z, ~ U S P, ~ S a ̃ o ~ P a u l o, ~ S P, ~ B r a s i l . ~}$ 


\section{INTRODUÇÃO}

Em 2004, o rebanho nacional de avestruzes era de 170.000 animais e, em 2005, o número aumentou para 335.425, representando uma taxa de crescimento de 97\% (MUNIZ, 2006). Este avanço na estrutiocultura brasileira requer do profissional médico veterinário maiores conhecimentos nesta área, mas a escassez de literatura ainda é um agravante para a solução dos problemas na criação, fato este relacionado ao rápido desenvolvimento dessa cultura em um curto período de tempo. Na rotina dos criatórios, são realizados diversos procedimentos para a manutenção da sanidade das aves, assim o uso de agentes anestésicos injetáveis possibilita a imobilização e manipulação dos animais, diminuindo a ocorrência de estresse e o risco de acidentes. Entretanto, ainda há poucos relatos de protocolos anestésicos para a contenção desta espécie e particularidades que possam interferir na administração dos fármacos.

Um aspecto da morfologia das aves muito discutido, porém com grandes divergências de sua importância, é a presença do sistema porta renal. As aves domésticas apresentam este sistema, constituído pelas veias portais renais craniais e caudais, que conduzem sangue para a divisão cranial e para as divisões média e caudal dos rins, respectivamente (KING, 1986). Na veia ilíaca comum, formada pela confluência das veias ilíaca externa e porta renal caudal, encontra-se, medialmente, na sua primeira porção, a valva porta renal (BAUMEL, 1986). Esta valva pode estar aberta ou fechada e este mecanismo parece depender, respectivamente, da atividade das fibras adrenérgicas ou colinérgicas presentes na mesma (AKESTER \& MANN, 1969; BENNETT \& MALMFORS, 1975; BURROWS et al., 1983). Portanto, quando a valva fecha uma maior parte do fluxo sanguíneo venoso dos membros pélvicos, este pode ser direcionado para o parênquima renal (BURROWS et al., 1983; CRUZ et al., 2001).

Há poucos relatos da presença do sistema porta renal em avestruzes (BEZUIDENHOUT, 1999, CORNICK-SEAHORN, 1996; FOWLER, 1991; HUCHZERMEYER, 1998; OELOFSEN, 1977) e estes não levam em consideração o local de aplicação dos agentes anestésicos com relação aos efeitos obtidos pelos mesmos, apesar da citação da circulação porta renal como um sistema que possivelmente afete os fármacos administrados nos músculos dos membros pélvicos. Desta forma, objetivou-se com este estudo caracterizar a anatomia do sistema porta renal e comparar sua influência sobre o protocolo anestésico xilazina, tiletamina e zolazepam quando administrado nos músculos da perna ou nos músculos da base da asa em avestruzes.

\section{MATERIAL E MÉTODOS}

Para a realização do estudo anatômico, utilizaram-se seis avestruzes (Struthio camelus, variedade domesticus), três machos e três fêmeas, com sete a 12 meses de idade, pesando entre 15 e 65kg. Após o procedimento de eutanásia, com sobredose anestésica de $30 \mathrm{mg} \mathrm{kg}^{-1}$ de tiopental sódico ${ }^{\mathrm{a}}$, realizouse, em cinco animais, a identificação, dissecação e canulação das veias femorais no sentido de drenagem. Em seguida, foi injetada solução aquosa corada de látex ${ }^{\mathrm{b}}$, utilizando-se seringa descartável e sob pressão manual moderada até a visualização dos vasos repletos. Posteriormente as peças foram fixadas, mediante a aplicação subcutânea, intramuscular e intracavitária de solução aquosa de formol ${ }^{\mathrm{c}}$ a $10 \%$, sendo, em seguida, imersas em recipientes contendo a mesma solução por no mínimo 72 horas. Os exemplares foram dissecados e, em seguida, retiraram-se os rins para visualização dos componentes do sistema porta renal. Em uma das aves, após a eutanásia, procedeu-se à retirada dos rins para a localização, colheita e fixação das valvas portais renais em formol a 10\%. Para a padronização dos termos anatômicos utilizados nas descrições dos resultados, empregou-se a Nômina Anatômica Avium (BAUMEL et al., 1993). As dissecações foram acompanhadas pela tomada de imagens fotográficas e elaboração de esquemas representativos, visando à ilustração e comprovação dos resultados.

Na contenção química, foram utilizados seis avestruzes (Struthio camelus, variedade domesticus), três machos e três fêmeas, com oito a 12 meses de idade, pesando entre 60 e 85kg, média 72,5 $\pm 9,35 \mathrm{~kg}$. Os animais foram capturados e posteriormente encapuzados, utilizando-se de gorros escuros. Após um período de repouso, realizou-se o exame préanestésico e, na seqüência, as aves foram pré-tratadas com xilazina ${ }^{\mathrm{d}}$ na dose de $1 \mathrm{mg} \mathrm{kg}^{-1} \mathrm{e}$, decorridos 10 minutos, receberam tiletamina/zolazepame na dose de $6 \mathrm{mg} \mathrm{kg} \mathrm{kg}^{-1}$. Os animais foram manipulados em duas ocasiões diferentes, sendo que na primeira anestesia o protocolo foi administrado nos músculos da base das asas (GI) e, após 15 dias, os mesmos animais receberam o protocolo nos músculos das pernas (GII).

Avaliou-se o período de latência, determinado por meio do tempo (minutos) compreendido entre a administração dos fármacos e a perda do tônus postural; o período hábil anestésico, considerado como o intervalo de tempo (minutos) em que o animal permaneceu completamente imobilizado 
até a primeira tentativa de endireitamento; e o período de recuperação total, considerado como o intervalo de tempo (minutos) desde a primeira tentativa de endireitamento até o retorno à posição bipedal. A indução e a recuperação foram classificadas em: boa, quando suave, rápida e sem riscos para o animal e para os assistentes; razoável, quando sem riscos para o animal e para os assistentes, mas com efeitos demorados ou período de transição ruim; e ruim, quando gerava perigo para o animal e assistentes ou ocorria excessiva agitação e necessidade de reaplicação do agente. Os parâmetros fisiológicos mensurados foram a freqüência cardíaca (FC), a freqüência respiratória (f) e a temperatura cloacal (TC). O relaxamento muscular foi avaliado subjetivamente por meio da resistência à flexão de membros e da observação de atividade muscular não induzida, segundo o escore proposto: 3 - intenso (flacidez muscular total); 2 - regular (discreto tônus muscular); 1 - leve (importante tônus muscular), e 0 - ausente (contrações durante manipulações). Os tempos de avaliação dos parâmetros fisiológicos foram: T0 - imediatamente antes da administração da MPA; T1 - 10 minutos após administração da MPA; T2 - 10 minutos após a administração da tiletamina/zolazepam; T3, T4 e T5 de 10 em 10 minutos após T2. Já o relaxamento muscular foi avaliado nos tempos após a administração da tiletamina/zolazepam.

Os períodos de latência, hábil e de recuperação dos grupos foram comparados estatisticamente pelo teste T de Student. Os parâmetros fisiológicos foram analisados utilizando o teste de Mann-Whitney, para comparar os grupos em cada tempo de avaliação, e o teste de Wilcoxon para comparar os tempos em cada grupo. As análises estatísticas foram realizadas em programa de computador $^{\mathrm{f}}$ e consideradas significativas quando $\mathrm{P}<0,05$. Os escores obtidos em relação à qualidade de indução, qualidade de recuperação e relaxamento muscular foram avaliados por freqüência relativa percentual de aparecimento nos grupos.

\section{RESULTADOS E DISCUSSÃO}

Nos resultados encontrados, evidenciou-se a presença de uma veia porta renal cranial e caudal em cada rim dos avestruzes, constituindo o sistema porta renal, conforme relatado em aves domésticas (BAUMEL et al., 1993; KING, 1986). Observou-se nas preparações que a veia femoral penetra na pelve cranialmente à articulação coxofemoral e ao passar, craniodorsalmente pelo tubérculo pré-acetabular (processo pectinal), torna-se conhecida como veia ilíaca externa, equivalente ao descrito por BAUMEL (1986) para a galinha. Dessa forma, o sangue proveniente do membro pélvico pode ser drenado para os rins a partir da união da veia ilíaca externa com a parte mais cranial da veia porta renal caudal (Figura 1). Os resultados mostram a veia isquiática demarcando as divisões média e caudal dos rins, onde também drena o sangue dos membros pélvicos para a veia porta renal caudal (Figura 1), mas não foi possível avaliar nas preparações a anastomose isquiofemoral (BAUMEL, 1986; BAUMEL et al., 1993).

A drenagem da veia mesentérica caudal na anastomose interilíaca e, conseqüentemente, na porção caudal da veia porta caudal renal (AKESTER, 1967; BAUMEL, 1986; BAUMEL et al., 1993), não foi evidenciada, já que apenas a origem das veias ilíacas internas estava visível nas preparações. No entanto, BEZUIDENHOUT (1999) afirmou que as veias da parede e vísceras pélvicas drenam para a veia ilíaca interna no avestruz e esta se une à veia porta renal caudal. A veia porta renal cranial localizou-se dorsalmente dentro do parênquima da divisão cranial do rim dos avestruzes, e assim como foi observado em patos por MAGRAS \& ASTERIADIS (1989), apresentou-se pouco desenvolvida, sendo evidente sua união à veia ilíaca comum (Figura 1) contudo, provavelmente devido ao seu tamanho reduzido, não foi possível visualizar a anastomose com o seio venoso vertebral interno (AKESTER, 1967; BAUMEL, 1986; BAUMEL et al., 1993). Portanto, nos avestruzes, não se caracterizou o anel venoso do sistema porta renal relatado por BAUMEL et al. (1993) e KING (1986) em aves domésticas.

A confluência da veia ilíaca externa com a veia porta renal caudal forma a veia ilíaca comum, que ainda recebe a veia renal caudal (BAUMEL, 1986; BAUMEL et al., 1993), fato observado também nos avestruzes. A união das veias ilíaca comum direita e esquerda forma a veia cava caudal, que se encontrou deslocada para a lateral direita em todas as preparações (Figura 1), assim a veia ilíaca comum esquerda mostrouse mais longa. O trajeto da veia porta renal caudal foi evidente na face dorsal dos rins somente entre as divisões craniais e caudais, já que o restante deste ocorre dentro do parênquima da divisão caudal até deixar os rins como veia ilíaca interna (Figura 1).

OELOFSEN (1977) descreveu a presença de três valvas portais renais, cônicas, em cada rim dos avestruzes, o que foi confirmado nos resultados encontrados, mas o autor as denominou de anterior, média e posterior. Nas observações do presente estudo e utilizando a Nomenclatura Anatômica Avium atual (BAUMEL et al., 1993), adotou-se a seguinte terminologia: valvas portais renais craniais, médias e 

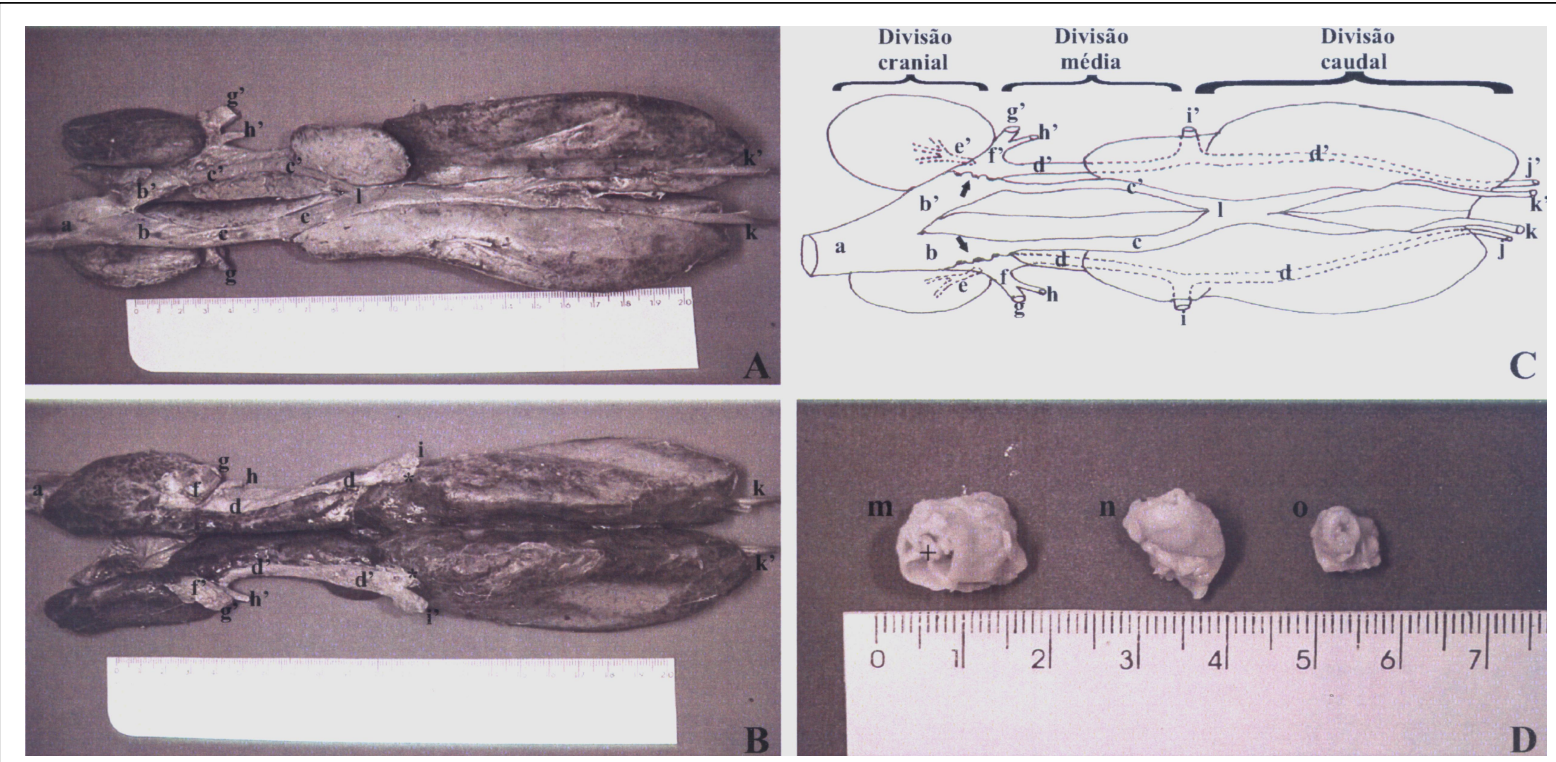

Figura 1 - A. Vista ventral dos rins e veias de avestruz. B. Vista dorsal dos rins e veias de avestruz. C. Desenho esquemático da vista ventral dos rins e veias de avestruz, evidenciando o trajeto dos vasos portais renais (linhas tracejadas). D. Valvas portais renais de avestruz. a-Veia cava caudal; b, b’-Veias ilíacas comuns direita e esquerda; c, c'-Veias renais caudais direita e esquerda; d, d'-Veias portais renais caudais direita e esquerda; e,e'-Veias portais renais craniais direita e esquerda; f, f'Veias ilíacas externas direita e esquerda; g, g'-Veias femorais direita e esquerda; h, h'-Veias púbicas direita e esquerda; i, i'-Veias isquiáticas direita e esquerda; j; j'-Veias ilíacas internas direita e esquerda; k, k'-Ureteres direito e esquerdo; lAnastomose entre as veias renais caudais; m-Valva porta renal cranial; n-Valva porta renal média; o-Valva porta renal caudal; * Ponto de entrada das veias portais renais caudais no parênquima renal; Setas ( $\rightarrow$ )-Localização das valvas portais renais; + Extensões das bordas papiladas dos óstios.

caudais. No pato e no ganso, elas são cônicas e possuem um óstio com borda irregularmente papilada (KING, 1986), o que também foi observado para as valvas portais renais craniais e médias dos avestruzes. Já as valvas portais renais caudais são geralmente menores e apresentam um formato mais cilíndrico semelhante às das alinhas (KING, 1986) (Figura 1). O ápice está voltado para a veia ilíaca comum e algumas valvas apresentaram extensões livres da borda do óstio irregular. Quanto à base, a disposição lado a lado das valvas forma uma parede contínua com a veia porta renal caudal (Figura 1).

Nas aves domésticas, há apenas uma valva em cada rim, que se localiza no início da veia ilíaca comum lateralmente à abertura da veia renal caudal e medialmente à confluência da veia porta renal caudal na veia ilíaca externa (BAUMEL, 1986; KING, 1986; MAGRAS \& ASTERIADIS, 1989). As valvas nos avestruzes foram localizadas na origem das veias ilíacas comuns, sendo que lateralmente observaram-se as veias portais renais craniais, veias ilíacas externas e veias portais renais caudais; enquanto medialmente encontram-se a união das veias renais caudais às veias ilíacas comuns (Figura 1). Assim, os resultados encontrados não corroboraram os de OELOFSEN
(1977), que descreveu a drenagem da veia isquiática para a região mais cranial da veia porta renal caudal, juntamente com as veias ilíaca externa e porta renal cranial, sendo que nas preparações ficou evidente a drenagem da veia isquiática no local onde a veia porta renal caudal penetra no parênquima renal da divisão caudal.

No procedimento anestésico, o período de

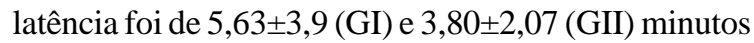
$(\mathrm{P}>0,05)$. Apesar de não haver diferença entre os grupos, os animais do GII apresentaram um tempo mais curto até a perda do tônus postural, provavelmente porque ocorreu absorção mais rápida dos fármacos, já que se trata de aves corredoras e há extensa vascularização nos músculos dos membros pélvicos. CRUZ et al. (2001) observaram o mesmo em papagaios que receberam quetamina nos músculos do peito. A qualidade de indução foi razoável em uma ave do GI (16,67\%), ruim em um animal (16,67\%) do GII e boa no restante das aves. CORNICK \& JENSEN (1992), CORNICK-SEAHORN (1996) e LIN et al. (1997), ao avaliarem a administração intravenosa de tiletamina/ zolazepam em avestruzes, relataram que a indução foi rápida e tranqüila, mas CORNICK \& JENSEN (1992) e CORNICK-SEAHORN (1996) também destacaram que 
a via intramuscular pode promover uma indução com excitação. Os resultados obtidos demonstraram que a administração intramuscular pode ser empregada, pois apenas uma ave apresentou sinais de excitação durante a indução.

Observou-se período hábil anestésico mais curto nas aves do GII (27,33 $\pm 9,75$ minutos), mas como não ocorreu diferença significativa quando se comparou ao GI (35,17 $\pm 8,13$ minutos), pode-se apenas sugerir que a influência da circulação porta renal em avestruzes foi de menor importância do que os relatos na literatura. Contudo, não se deve descartar que as diferenças no metabolismo desta espécie possam alterar a duração de ação da associação tiletamina/zolazepam. A maior duração da anestesia no GI pode estar relacionada à lenta absorção dos fármacos, já que os músculos na base das asas são pouco desenvolvidos e conseqüentemente bem menos vascularizados.

A recuperação demorou mais no GI (33,67 $\pm 10,20$ minutos), mas a diferença não foi

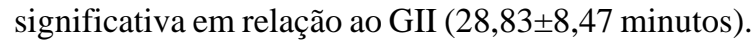
Assim, deve-se considerar a velocidade de absorção dos fármacos anestésicos nos grupos e talvez menor interferência do desvio porta renal no GII. Em relação à avaliação da qualidade de recuperação, os resultados obtidos foram similares em ambos os grupos, o que pode sugerir uma característica individual quanto à farmacocinética do protocolo anestésico empregado, principalmente na sua metabolização. As aves com recuperação ruim (16,67\%) e razoável (16,67\%) apresentaram hipertonia muscular, movimentos de pedalar e movimentação da cabeça e pescoço, sendo mais pronunciados em um animal com escore ruim nas duas anestesias. Esses efeitos podem ser minimizados com o uso do diazepam na recuperação (CORNICK \& JENSEN, 1992; CORNICK-SEAHORN, 1996; GILSLEIDER, 1998; LIN et al., 1997; PERELMAN, 1999) ou da azaperona no momento da indução (PERELMAN, 1999), já que o zolazepam parece ser biotransformado mais rápidamente que a tiletamina nos avestruzes (CORNICK \& JENSEN, 1992).

Em relação ao protocolo anestésico, ocorreu redução na freqüência cardíaca após a administração da xilazina (T1), sendo estatisticamente significativa quando se comparou aos valores basais (T0) (Tabela 1). Este efeito também foi relatado por CORNICK \& JENSEN(1992); CORNICK-SEAHORN(1996); CULLEN et al. (1995); GANDINI et al. (1986) e LIN et al. (1997). Assim, a partir dos resultados obtidos e de acordo com LUDDERS \& MATTHEUS (1996), deve-se evitar o emprego deste fármaco em aves debilitadas, e ainda esta depressão cardiovascular pode ser potencializada com a anestesia inalatória (CORNICK-SEAHORN, 1996; LIN et al., 1997). Nos tempos após a aplicação da

Tabela 1 - Mediana e quartis da freqüência cardíaca (FC) (batimentos/minuto), freqüência respiratória (f) (movimentos respiratórios/minuto) e temperatura cloacal (TC) (graus Celsius) dos avestruzes tratados com xilazina, tiletamina e zolazepam na musculatura da asa (GI) ou da perna (GII) nos diversos tempos de avaliação.

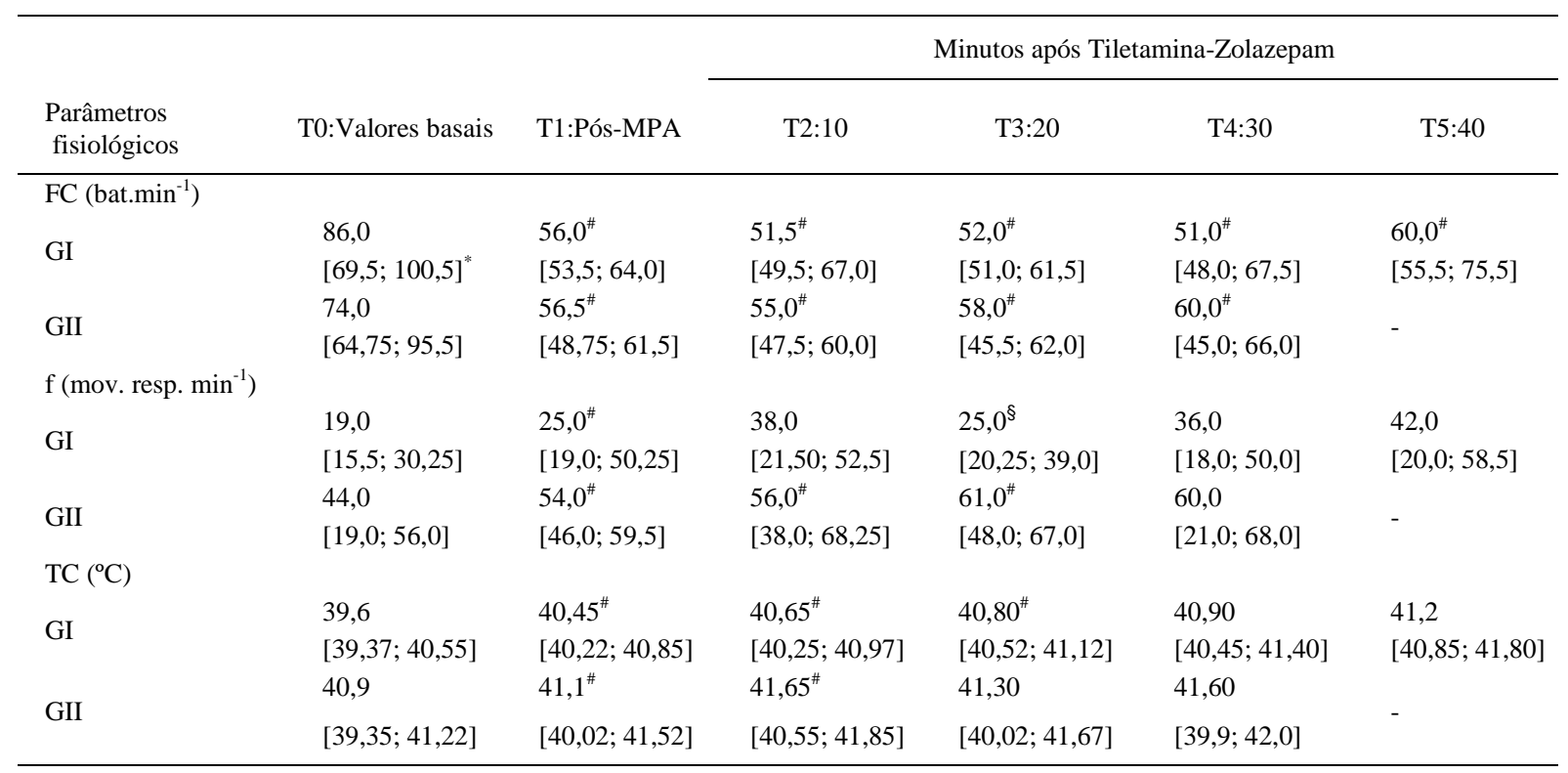

MPA - medicação pré-anestésica.

*os valores entre colchetes representam o primeiro e o terceiro quartis, respectivamente.

${ }^{\#} \mathrm{P}<0,05$ em relação aos valores basais (T0); ${ }^{\S} \mathrm{P}<0,05$ entre os grupos I e II. 
tiletamina/zolazepam, a freqüência cardíaca permaneceu abaixo dos valores basais (T0) nos dois grupos $(\mathrm{P}<0,05)$ (Tabela 1$)$, conforme também foi relatado por LIN et al. (1997), que empregaram xilazina e butorfanol na medicação pré-anestésica. Diferentemente, VAN HEERDEN \& KEFFER (1991), além de não observarem bradicardia com o uso isolado da tiletamina/zolazepam, obtiveram incremento da freqüência cardíaca após a dose de $20 \mathrm{mg} \mathrm{kg}^{-1}$, i.m.

Nos dois grupos ocorreu elevação da temperatura cloacal, sendo o maior incremento nos animais do GII (Tabela 1). De acordo com HUCHZERMEYER (1998), as temperaturas normais registradas em avestruzes variam de 37,8 a $38,9^{\circ} \mathrm{C}$, atingindo até mesmo $40,7^{\circ} \mathrm{C}$. No entanto, dentre os valores basais obtidos no presente estudo, registrouse a temperatura de $41,3^{\circ} \mathrm{C}$. A temperatura corporal nas avestruzes é regulada pela variação no posicionamento das plumas e o aumento da freqüência respiratória, ou arquejamento, que possibilita a perda de calor por evaporação a partir da traquéia, dos sacos aéreos e da faringe(ELMÔR et al., 2004; HUCHZERMEYER, 1998). Dessa forma, além da temperatura ambiental elevada, o decúbito esternal e a imobilização dos animais dificultaram a perda de calor, o que justifica o incremento da temperatura cloacal nos grupos.

O incremento da freqüência respiratória, observado na maioria dos animais, principalmente no GII (Tabela 1), demonstrou que a via intramuscular permite uma indução anestésica sem a ocorrência de apnéia, já que LIN et al. (1997) e CORNICK \& JENSEN (1992) observaram este efeito após a aplicação intravenosa da tiletamina/zolazepam e manutenção com isofluorano. Em T3, alguns animais do GI apresentaram redução da freqüência respiratória, sendo mais evidente em uma ave, justificando, assim, a diferença estatística encontrada ao comparar com o GII (Tabela 1). Essa observação parece estar relacionada à absorção mais lenta dos anestésicos neste grupo e com o maior período de imobilização.

Apenas dois animais (33,33\%) do GI apresentaram relaxamento muscular intenso e com duração aproximada de 20 minutos, enquanto no GII o mesmo grau de miorrelaxamento ocorreu em cinco aves (83,33\%), mas por 10 a 15 minutos. Portanto, pode ser necessária uma dose mais elevada da tiletamina/ zolazepam, quando for administrada nos músculos da base da asa, já que a lenta absorção parece ter disponibilizado menor quantidade dos fármacos para os seus locais de ação, dificultando o estabelecimento de uma anestesia com completo miorrelaxamento em quatro animais. No GII, a absorção mais rápida promoveu relaxamento muscular intenso na maioria das aves, apesar da curta duração, o que pode estar relacionado à metabolização e à excreção de parte dos fármacos em decorrência do desvio porta renal ou por ambos.

\section{CONCLUSÕES}

Diante da metodologia empregada, concluise que o arranjo anatômico dos vasos e das valvas do sistema porta renal desvia parte do fluxo sanguíneo venoso proveniente dos membros pélvicos para os rins. No entanto, o protocolo anestésico administrado nos músculos das pernas promove contenção química adequada para a realização de procedimentos de curta duração a campo nos avestruzes.

\section{AGRADECIMENTOS}

À Coordenação de Aperfeiçoamento de Pessoal de Nível Superior (CAPES) e aos criatórios Vereda dos Avestruzes, Império dos Avestruzes e War Ranch.

\section{FONTES DE AQUISIÇÃO}

aThionembutal - Abbot.

bLátex natural centrifugado 60\% - Látex Altamira.

'Formol 37\% comercial - Allkimia.

dAnasedan - Vetbrands.

eZoletil 50 - Virbac.

${ }^{\mathrm{f}}$ Software Minitab 14

\section{COMITÊ DE ÉTICA}

Este estudo foi aprovado pela Comissão de Bioética da Faculdade de Medicina Veterinária e Zootecnia da Universidade de São Paulo (FMVZ-USP), sob o protocolo número 487/2004.

\section{REFERÊNCIAS}

AKESTER, A.R. Renal portal shunts in the kidney of the domestic fowl. J Anat, v.101, n.3, p.569-594, 1967.

AKESTER, A.R.; MANN, S.P. Adrenergic and cholinergic innervation of the renal portal valve in the domestic fowl. $\mathbf{J}$ Anat, v.104, n.2, p.241-252, 1969.

BAUMEL, J.J. Coração e vasos sanguíneos das aves. In: GETTY, R. Sisson \& Grossman: anatomia dos animais domésticos. 5.ed. Rio de Janeiro: Guanabara Koogan, 1986. V.2, p.1842-1889.

BAUMEL, J.J. et al. Handbook of avian anatomy: nomina anatomica avium. 2.ed. Cambridge: Berge, 1993. 779p.

BENNETT, T.; MALMFORS, T. Autonomic control of renal portal blood flow in the domestic fowl. Experientia, v.31, n.10, p.1177-1178, 1975

BEZUIDENHOUT, A.J. Anatomy. In: DEEMING, D.C. The ostrich: biology, production and health. London: CABI, 1999. p.13-49.

BURROWS, M.E. et al. Avian renal portal valve: a reexamination of its innervation. Am J Physiol, v.245, n.4, p.H628-634, 1983.

Ciência Rural, v.37, n.6, nov-dez, 2007. 
CORNICK, J.L.; JENSEN, J. Anesthetic management of ostriches. J Am Vet Med Assoc, v.200, n.11, p.1661-1666, 1992.

CORNICK-SEAHORN, J.L. Anesthesiology of ratites. In: SHANE, S.M.; TULLY, T.N. Ratite management, medicine, and surgery. Florida - Malabar: Kriegler, 1996. p.79-94.

CRUZ, M.L. et al. Avaliação da importância do sistema portarenal em papagaios (Amazona aestiva). Rev Educ Contin CRMV-SP, v.4, n.1, p.40-44, 2001.

CULLEN, L.K. et al. Ostrich anaesthesia: xylazine premedication followed by alphaxalone/alphadolone and isoflurano. Aust Vet J, v.72, n.4, p.153-154, 1995.

ELMÔR, R.A. et al. Biologia e meio ambiente. In: CARRER, C.C. et al. A criação do avestruz: guia completo de A a Z. Pirassununga: C.C. Carrer, 2004. p.17-32.

FOWLER, M.E. Comparative clinical anatomy of ratites. J Zoo Wildl Med, v.22, n.2, p.204-227, 1991.

GANDINI, G.C.M. et al. An anaesthetic combination of ketamine, xylazine and alphaxalone-alphadolone in ostriches (Struthio camelus). Vet Rec, v.118, n.26, p.729-730, 1986.

GILSLEIDER, E.F. Anesthesia and surgery of ratites. Vet Clin North Am Food Anim Pract, v.14, n.3, p.503-524, 1998.

HUCHZERMEYER, F.W. Diseases of ostriches and other ratites. Onderstepoort: Agricultural research council, 1998. 296p.
KING, A.S. Aparelho urogenital das aves. In: GETTY, R. Sisson \& Grossman: anatomia dos animais domésticos. 5.ed. Rio de Janeiro: Guanabara Koogan, 1986. V.2, p.1798-1839.

LIN, H.C. et al. Use of xylazine, butorfanol, tiletaminezolazepam, and isoflurane for induction and maintenance of anesthesia in ratites. J Am Vet Med Assoc, v.210, n.2, p.244248, 1997.

LUDDERS, J.W.; MATTHEUS, N. Birds. In: THURMON, J.C. et al. Lumb \& Jones' veterinary anesthesia. 3.ed. Baltimore: Willians \& Wilkins, 1996. p.645-669.

MAGRAS, I.N.; ASTERIADIS, G.I. Anatomical study of the renal portal system in the duck (Anas platyrhincos). Arch Anat Histol Embryol, v.72, p.105-111, 1989.

MUNIZ, L.R. Estatística do atual rebanho de avestruzes no Brasil. In: ACAB. Anuário da estrutiocultura brasileira 2005/06. São Paulo: ACAB, 2006. p.107-112.

OELOFSEN, B.W. The renal portal valves of the ostrich, Struthio camelus. S Afr J of Sci, v.73, p.57-58, 1977.

PERELMAN, B. Health management and veterinary procedures. In: DEEMING, D.C. The ostrich: biology, production and health. London: CABI, 1999. p.321-346.

VAN HEERDEN, J.; KEFFEN, R.H. A preliminary investigation into the immobilizing potential of a tiletamine/zolazepam mixture, metomidate, a metomidate and azaperone combination and medetomidine in ostriches (Struthio camelus). J S Afr Vet Assoc, v.62, n.3, p.114-117, 1991. 